OPEN ACCESS

Edited by:

Emily Keshner,

Temple University, United States

Reviewed by:

Tyler Fettrow,

University of Florida, United States

Ashwini K. Rao,

Columbia University, United States

*Correspondence:

Florian S. Eichler feichler@partners.org

Specialty section:

This article was submitted to Movement Disorders, a section of the journal

Frontiers in Neurology

Received: 22 March 2021

Accepted: 24 May 2021

Published: 17 June 2021

Citation:

Godbole NP, Sadjadi R, DeBono MA,

Grant NR, Kelly DC, James PF,

Stephen CD, Balkwill MD, Lewis RF

and Eichler FS (2021) Gait Difficulties

and Postural Instability in

Adrenoleukodystrophy.

Front. Neurol. 12:684102.

doi: 10.3389/fneur.2021.684102

\section{Gait Difficulties and Postural Instability in Adrenoleukodystrophy}

\author{
Neha P. Godbole ${ }^{1}$, Reza Sadjadi ${ }^{1,2}$, Madeline A. DeBono ${ }^{1}$, Natalie R. Grant ${ }^{1}$, \\ Daniel C. Kelly ${ }^{1}$, Peter F. James ${ }^{1}$, Christopher D. Stephen ${ }^{1,2}$, M. David Balkwill ${ }^{3}$, \\ Richard F. Lewis ${ }^{2,3}$ and Florian S. Eichler ${ }^{1,2 *}$ \\ ${ }^{1}$ Department of Neurology, Massachusetts General Hospital, Boston, MA, United States, ${ }^{2}$ Harvard Medical School, Boston, \\ MA, United States, ${ }^{3}$ Massachusetts Eye and Ear, Boston, MA, United States
}

Background: Gait and balance difficulties are among the most common clinical manifestations in adults with X-linked adrenoleukodystrophy, but little is known about the contributions of sensory loss, motor dysfunction, and postural control to gait dysfunction and fall risk.

Objective: To quantify gait and balance deficits in both males and females with adrenoleukodystrophy and evaluate how environmental perturbations (moving surfaces and visual surrounds) affect balance and fall risk.

Methods: We assessed sensory and motor contributions to gait and postural instability in 44 adult patients with adrenoleukodystrophy and 17 healthy controls using three different functional gait assessments (25 Foot Walk test, Timed Up and Go, and 6 Minute Walk test) and computerized dynamic posturography.

Results: The median Expanded Disability Status Scale score for the patient cohort was 3.0 (range 0.0-6.5). Both males and females with adrenoleukodystrophy showed impairments on all three functional gait assessments relative to controls $(P<0.001)$. Performance on walking tests and Expanded Disability Status Scale scores correlated with incidence of falls on computerized dynamic posturography, with the 25 Foot Walk being a moderately reliable predictor of fall risk (area under the ROC curve $=0.7675$, $P=0.0038)$.

Conclusion: We demonstrate that gait difficulties and postural control deficits occur in patients with adrenoleukodystrophy, albeit at an older age in females. Postural deficits were aggravated by eyes closed and dynamic conditions that rely on vestibular input, revealing challenges to the interplay of motor, sensory and vestibular circuitry in adrenoleukodystrophy.

Keywords: adrenoleukodystrophy, balance, myelopathy, spinal cord disease, gait, computerized dynamic posturography

\section{INTRODUCTION}

$\mathrm{X}$-linked adrenoleukodystrophy (ALD) is a neurogenetic disorder of brain and spinal cord caused by mutations in the $A B C D 1$ gene, which result in decreased levels of $A B C D 1$, a membrane protein that transports very long chain fatty acids into peroxisomes for degradation (1). In adulthood, ALD manifests as a non-inflammatory myelopathy referred to as adrenomyeloneuropathy (2-4), 
but patients remain at risk for developing brain inflammation characteristic of childhood $\operatorname{ALD}(5,6)$. Brain inflammation is seen in males with cerebral ALD but rarely in female heterozygotes. While this most commonly leads to progressive neurological decline, active inflammation can also resolve and stabilize as self-arrested cerebral ALD (7). It is clear that myelopathy affects these males with stable brain lesions as well (8). Patients suffer from gait and balance difficulties across the phenotypic spectrum in adulthood (9-11), but little is known about the relative contributions of sensory loss, motor dysfunction, and postural control to gait dysfunction and fall risk. Multidimensional quantitative performance measures are needed to better characterize and define the association between sensory deficits and balance issues in both males and females with ALD.

Computerized dynamic posturography (CDP) assesses standing balance using a moveable force plate to simulate conditions that mirror those encountered in daily life (12). Using this platform, sensory organization testing (SOT) contains a set of six conditions to evaluate a patient's ability to integrate somatosensory, visual, and vestibular inputs to minimize sway (13). This tool has been used to assess postural instability in Parkinson disease (14) and to evaluate balance in older adults (15). CDP may therefore be an ideal tool to better characterize and quantitate the myelopathy of ALD. We sought to perform quantitative postural measures to assess standing balance, compare these measures with functional gait assessments, and identify potential predictors of fall risk in males and females with ALD.

\section{MATERIALS AND METHODS \\ Design}

In this study, males and females over the age of 18 years with a diagnosis of ALD were recruited from the Leukodystrophy Clinic at Massachusetts General Hospital. All participants provided written informed consent and the study was approved by the local Institutional Review Board, the Mass General Brigham Human Research Committee. We included patients with genetically confirmed X-ALD, without radiographic evidence of active inflammatory disease on brain MRI (absence of lesional contrast enhancement). We excluded patients with the presence of other confounding neurological deficits that in the investigator's opinion could affect balance, being non-ambulatory (using a wheelchair), or an Expanded Disability Status Scale (EDSS) score of $\geq 7$ determined by neurological examination (16). Neurological examinations were conducted by two neurologists with expertise in neuromuscular disorders (F.E. and R.S.). We also enrolled age-matched healthy control participants age $\geq 18$ years.

\section{Retrospective Chart Review}

We retrospectively reviewed medical charts of patients who attended the Massachusetts General Hospital Leukodystrophy

Abbreviations: ALD, X-linked Adrenoleukodystrophy; AUC, Area Under the Curve; CDP, Computerized Dynamic Posturography; EDSS, Expanded Disability Status Scale; ROC, Receiver-Operating Characteristic; SOT, Sensory Organization Testing; 25FW, 25 Foot Walk; TUG, Timed Up and Go; 6MW, 6 Minute Walk.
Clinic from November 2017 to September 2019 and yielded 44 patients with ALD, of which three were excluded due to incomplete clinic notes. We extracted data on the presence of seven major neurological signs and symptoms including neuropathic pain, lower extremity sensory loss, gait disorder, use of a walking aid, balance difficulty, urinary urgency, fecal incontinence, and erectile dysfunction (in male patients). Balance difficulty was identified by either patient complaint and/or a positive Romberg sign documented in the clinic note during the neurological examination. These data were obtained in order to assess the extent of myelopathy-associated signs and symptoms with which our patient cohort presented.

We also recorded the presence of lesions as evident on brain MRIs performed as standard of care. The brain MRIs encompassed axial T2-weighted as well as pre- and post-contrast T1-weighted sequences. Non-inflammatory lesions were defined as lesions that did not show contrast enhancement on the postcontrast T1-weighted sequence. Active inflammatory lesions were defined as those displaying contrast enhancement. These patients with active inflammation were excluded from our study.

\section{Functional Gait Assessment}

We assessed participants' gait disability using three functional outcome measures: the 25 Foot Walk (25FW) test, the Timed Up and Go (TUG) walking test, and the 6 Minute Walk (6MW) test. All walking tests were performed in a flat, unobstructed $30 \mathrm{~m}$ walkway. Start and finish lines for each of the tests were clearly marked and the average of two trials was recorded for both the 25FW and TUG. For the TUG, participants were instructed to rise from a chair without using the arm rests, walk $10 \mathrm{ft}$, pivot, and return to the chair again without using the arm rests for support. The time from when the participant arose from the chair and when they sat back down was recorded.

\section{Quantitative Balance Testing}

We analyzed balance using the NeuroCom SMART Equitest posturography platform $(13,14)$ to assess postural sway, a proxy for standing balance. Standardized instructions were provided to all patients prior to testing. As a safety precaution, participants were strapped into a modified parachute harness prior to testing and a gait belt was used for additional support as necessary. Balance testing included the sensory organization testing (SOT), which measures the amount of sway as an equilibrium score from 0 to 100, with 100 being perfect stability and 0 representing a fall. Individuals without balance impairments can tolerate anterior/posterior sway of 12.5 degrees without falling. The equilibrium score is calculated by comparing the participant's anterior/posterior sway to the 12.5 degree maximum. Sway angles were computed based on the participant's center of gravity at an upright stance and during testing (17). The SOT captures both static and dynamic balance through six conditions in the following order:

1. Eyes open, stable surface, stable visual surround.

2. Eyes closed, stable surface, stable visual surround.

3. Eyes open, stable surface, sway-referenced visual surround.

4. Eyes open, sway-referenced surface, stable visual surround. 
5. Eyes closed, sway-referenced surface, stable visual surround.

6. Eyes open, sway-referenced surface, sway referencedvisual surround.

The software reports individual scores for each condition and a composite equilibrium score based on the participant's performance across all six conditions. Three $20 \mathrm{~s}$ trials of each condition were conducted and best performance across the trials was used for analysis. We were particularly interested in assessing conditions that remove visual cues to unmask the proprioceptive deficits known to occur in patients with ALD. Falls during SOT were defined as losing balance and being caught by the safety harness, taking a step off of the platform, or using the walls of the machine for stability, similar to the falls criteria used in previous studies (14). To capture the potential for fall risk, the number of falls patients experienced during the SOT were compared to their performances on the functional gait assessments and EDSS score. Comparisons between groups were performed for each condition separately and for the composite equilibrium score. If a participant fell on all three trials of a testing condition, a maximum score could not be obtained, and they were excluded in the analysis involving that condition.

\section{Statistical Analysis}

All statistical tests were performed in GraphPad Prism version 8.0.0 (San Diego, CA, USA) and significance was designated as two-tailed $P<0.05$ with Bonferroni correction for multiple testing. Non-parametric testing with Bonferroni correction for multiple testing, including Mann-Whitney $U$-test, was used to compare the following groups: patients and healthy controls, male and female patients, patients who did not fall during SOT and patients who did fall during SOT.

Binormal receiver-operating characteristic (ROC) curves were generated for each of the gait assessments, (6MW, TUG, $25 \mathrm{FW}$ ) by SOT falls. This was done to assess the capability of these measures to predict fall risk and determine cutoff values. Lastly, we used Spearman rank-order correlation to analyze the association between performance on clinical outcome measures and SOT scores as well as the number of falls during SOT.

\section{RESULTS}

\section{Clinical Description and Demographics}

We enrolled 44 adult patients with ALD (22 male, 22 female) and 17 healthy control participants (nine male, eight female). Descriptive characteristics of 41 of 44 participants in the patient cohort are listed in Table 1. Thirteen of the 22 male patients had radiographic evidence of non-inflammatory white matter disease. The median age of the total cohort was 42 (range 19-77) years. Males were 37.5 (IQR 23-41.25) years and females were older at 52 (IQR 44.5-58.5) years $(P<0.001)$. The two most common symptoms experienced were lower extremity sensory disturbance and balance difficulty, both affecting 32 of $41(78.0 \%)$ of patients. Of the 29 patients with a gait disorder, $11(26.8 \%$ of total cohort) utilized a walking aid. Seven of 22 males $(31.8 \%)$ reported erectile dysfunction. Based on a limited subset of patient data $(n=20)$ available from chart review, the median age of myelopathy onset was 30 (range 18-63) years. The median EDSS score for the total cohort was 3.0 (range 0.0-6.5). A score of 3 indicates a combination of moderate and mild disability in different functional systems while being fully ambulatory.

\section{Functional Gait Assessments}

All 44 participants enrolled completed the following three functional gait assessments: $6 \mathrm{MW}$, TUG, $25 \mathrm{FW}$. Compared to controls, patients with ALD had significantly shorter median 6MW distances [454 (IQR 348-515) m vs. 569 (IQR 513-628) $\mathrm{m}, P<0.0001$ ], and longer median times for TUG [8.33 (IQR 6.97-11.57) s vs. 5.78 (IQR 5.24-6.73) s, $P<0.0001]$ and 25FW [5.62 (IQR 4.83-6.74) s vs. 4.11 (IQR 3.77-4.84) s, $P<$ 0.0001] (Table 2). Additionally, stratifying the patient cohort by sex showed no significant difference between males and females (6MW: $P=0.3483$, TUG: $P=0.0686,25 \mathrm{FW}: P=0.1847$ ).

\section{Quantitative Balance Testing}

Results for CDP SOT by disease status are shown in Table 3. Significant differences were found between the patient and healthy control cohorts for the composite equilibrium score and all conditions $(P<0.01)$ except for condition four (eyes open, dynamic surface, stable visual surround). No significant differences were found between males and females (SOT overall $P=0.4595)$. Likewise, no significant differences were found between males with and without non-inflammatory lesions (SOT overall $P=0.9870)$. There was a weak negative relationship between SOT score and 25FW $(r=-0.3797, P=0.0110)$, and a moderate negative relationship between SOT and EDSS scores $(r$ $=-0.5574, P=<0.001)$. Whereas, higher SOT scores represent better function, higher $25 \mathrm{FW}$ and EDSS scores represent more severe disability.

\section{Falls Risk Assessment}

Falls data were compared in two ALD groups, with patients divided into categories of "no falls" or "falls" depending on what they experienced during the balance testing. Most SOT falls occurred during conditions five or six (91.9\%). Of the 23 patients who fell during SOT, 21 fell only on conditions five or six $(91.3 \%)$. Differences were found between the no falls and falls groups for all three walking assessments and EDSS score (Table 4). Compared to the no falls group, the falls group had a shorter median 6MW distance [366 (IQR 297-502) m vs. 476 (IQR 426-569) m, $P=0.0089$ ], and longer median times for TUG [9.12 (IQR 7.57-13.02) s vs. 7.55 (IQR 6.55-8.56) s, $P=$ 0.0126 ] and 25FW [6.51 (IQR 5.28-8.59) s vs. 4.94 (IQR 4.715.97) s, $P=0.0012]$. The falls group also had higher EDSS scores [3.5 (IQR 2.5-4.0) vs. 2.0 (IQR 0.5-3.0), $P=0.0020$ ]. ROC curves were generated for falls in each of the functional gait assessments. The $25 \mathrm{FW}$ test had the highest area under the curve (AUC) value, 0.7675 (IQR 0.6192-0.9158), while the TUG test had the lowest, 0.7100 (IQR 0.5459-0.8741). The cutoff values for each assessment were $373.4 \mathrm{~m}, 8.345 \mathrm{~s}$, and $5.0 \mathrm{~s}$ for the $6 \mathrm{MW}, \mathrm{TUG}$, and 25FW test, respectively (Table 5). There were weak correlations between falls on SOT and clinical outcome 
TABLE 1 | Symptoms present on neurological examination and EDSS scores for the patient cohort stratified by age and sex.

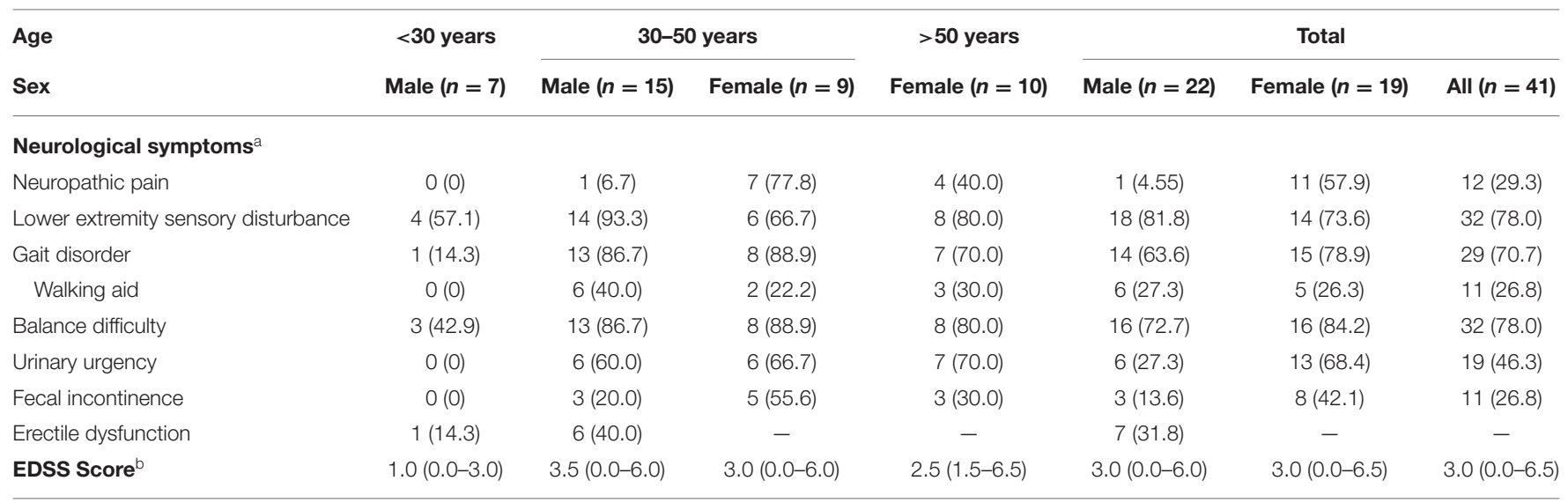

EDSS, Expanded Disability Status Scale; $n$, number of participants.

${ }^{a}$ Symptoms are presented as absolute numbers (percentage).

${ }^{b}$ EDSS scores are presented as median (range).

TABLE 2 | Performance on gait assessment outcomes for patients and controls.

\begin{tabular}{|c|c|c|c|c|}
\hline Outcome measure & $\operatorname{ALD}(n=44)^{\mathrm{a}}$ & Control $(n=17)^{\mathrm{a}}$ & Test statistic $^{b}$ & $P$-value \\
\hline TUG (s) & $8.33(6.97-11.57)$ & $5.78(5.24-6.73)$ & 111.5 & $<0.0001^{\star \star}$ \\
\hline
\end{tabular}

ALD, Adrenoleukodystrophy; $n$, number of participants; 6MW, 6 Minute Walk; TUG, Timed Up and Go; 25FW, 25 Foot Walk; IQR, Interquartile Range.

a Scores are reported as median (IQR).

${ }^{b}$ Differences between patient and control scores were assessed with Mann-Whitney U-tests.

${ }^{\star \star} P<0.01$.

TABLE 3 | Performance on SOT for patients and controls.

\begin{tabular}{|c|c|c|c|c|c|}
\hline & $n$ & $\operatorname{ALD}(n=44)^{\mathrm{a}}$ & Control $(n=17)^{a}$ & Test statistic $^{b}$ & $P$-value \\
\hline SOT condition 1 & 44 & $94(92-96)$ & $97(96-97)$ & 99 & $<0.0001^{\star \star}$ \\
\hline SOT condition 2 & 43 & $90(85-94)$ & $95(94-96)$ & 118 & $<0.0001^{\star *}$ \\
\hline SOT condition 3 & 44 & $91(86-94)$ & $95(93-96)$ & 141 & $<0.0001^{\star *}$ \\
\hline SOT condition 4 & 44 & $85(79-88)$ & 87 (84-93) & 248.5 & 0.0428 \\
\hline SOT condition 5 & 40 & 66 (56-72) & 79 (74-82) & 113.5 & $<0.0001^{\star *}$ \\
\hline
\end{tabular}

ALD, Adrenoleukodystrophy; SOT, Sensory Organization Testing; n, number of participants; IQR, Interquartile Range.

${ }^{\text {a }}$ Scores are reported as median (IQR).

${ }^{b}$ Differences between patient and control scores were assessed with Mann-Whitney U-tests.

${ }^{\star *} P<0.01$.

measures, with the strongest relationships being between the 25FW and EDSS score $(r=0.4980, P=0.0006$ and $r=0.4976$, $P=0.0006$, respectively).

\section{DISCUSSION}

In this study, we used functional gait assessments and quantitative balance testing to demonstrate impairments of gait, balance, and posture in adults with ALD. Importantly, CDP revealed meaningful deficits in postural control in both males and females. These findings were associated with a higher incidence of falls during balance testing and did not correlate with presence of non-inflammatory brain lesions on MRI.

Our findings in females with ALD are congruent with prior studies and cannot be explained merely by the older median age of females compared with males in our cohort. A previous study of leg strength, tone, and vibration in patients with ALD found that sex differences were more pronounced in the earlier "mild" form of disease, but disease severity was comparable between males and females in the "moderate" and "severe" stages 
TABLE 4 | Performance on gait assessments and EDSS scores for patients who did and did not fall on balance testing.

\begin{tabular}{|c|c|c|c|c|}
\hline Outcome measure & No falls $(n=21)^{\mathrm{a}}$ & Falls $(n=23)^{\mathrm{a}}$ & Test statistic $^{b}$ & $P$-value \\
\hline 6MW (m) & 476 (426-569) & 366 (197-502) & 131.5 & $0.0089^{\star *}$ \\
\hline TUG (s) & 7.55 (6.55-8.56) & $9.12(7.57-13.02)$ & 136 & $0.0126^{\star}$ \\
\hline $25 F W(s)$ & $4.94(4.71-5.97)$ & 6.51 (5.28-8.59) & 107 & $0.0012^{\star \star}$ \\
\hline EDSS score & $2.0(0.5-3.0)$ & $3.5(2.5-4.0)$ & 114 & $0.0020^{\star \star}$ \\
\hline
\end{tabular}

n, number of participants; 6MW, 6 Minute Walk; TUG, Timed Up and Go; 25FW, 25 Foot Walk; IQR, Interquartile Range.

a Scores are reported as median (IQR).

${ }^{b}$ Differences between fallers and non-fallers scores were assessed with Mann-Whitney U-tests.

${ }^{\star} P<0.05$.

${ }^{\star *} P<0.01$.

TABLE 5 | ROC analysis for predicting falls during SOT in patients with ALD.

\begin{tabular}{|c|c|c|c|c|c|}
\hline Outcome measure & Cutoff value & AUC $(95 \% \mathrm{Cl})$ & Sensitivity (\%) & Specificity (\%) & $P$-value \\
\hline $6 \mathrm{MW}(\mathrm{m})$ & 373.4 & $0.7063(0.5434-0.8691)$ & 50 & 90 & $0.0256^{*}$ \\
\hline TUG (s) & 8.345 & $0.7100(0.5459-0.8741)$ & 65 & 80 & $0.0231^{*}$ \\
\hline 25FW (s) & 5.0 & $0.7675(0.6192-0.9158)$ & 90 & 60 & $0.0038^{\star \star}$ \\
\hline
\end{tabular}

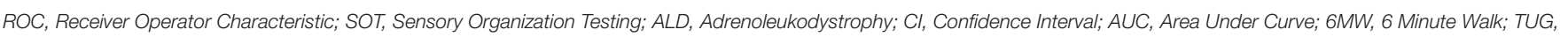
Timed Up and Go; 25FW, 25 Foot Walk.

${ }^{*} P<0.05$.

${ }^{\star \star} P<0.01$.

of disease (9). Our findings align with this study, suggesting that while females have later onset of symptoms, they may eventually suffer from a similar disease burden as males.

Functional gait assessments and CDP SOT in males and females with ALD revealed impairments on all three walking tests, as well as proprioceptive deficits demonstrated by removing visual cues during SOT. Walking distance and speed were comparable to prior studies (10). In our study, 13 male patients had evidence of non-inflammatory lesions on MRI at least 1 year prior to testing. These patients showed no significant differences on quantitative performance measures to patients without brain lesions, suggesting that long-term compensation may have occurred or that the myelopathy alone is a larger determinant of performance.

Importantly, adult patients with ALD struggled to maintain balance on conditions five and six of CDP testing, as indicated by the high number of falls. These conditions have a dynamic platform and eliminate or minimize the use of visual compensatory mechanisms, either by directly having the patient close their eyes or by changing visual perception through the use of a moving surrounding. The magnitude of change in these conditions was far greater than with moving surface alone without perturbation of the visual surrounds (condition four). Similar to other diseases with prominent dorsal column/proprioceptive involvement, our findings suggest that patients with ALD rely more heavily on their vision to maintain balance in dynamic conditions. A majority of falls occurred in conditions five and six, which is comparable to studies in Parkinson disease (18). These conditions challenge vestibular function (19), which could suggest that impairments in postural control may be aggravated by vestibular dysfunction and not visual processing alone. Future studies should include standard assessments of vestibular function and cognitive-visual processing, including yaw axis sinusoidal rotation and video head impulse tests.

Of note, the outcomes on all three walking tests and EDSS scores correlated with falling during the SOT. Literature in Parkinson disease has shown that slower times on the TUG walking test are associated with self-reported fall frequency (20). In pursuit of a more rigorous metric than anecdotal reporting, we determined the number of falls in real time through SOT. The ROC analysis showed that all three walking tests had moderate discriminatory ability, with AUC values $>0.7$. Of the three gait assessments, the 25FW test was the most sensitive predictor of falls during SOT. One reason for the higher discriminatory value of the $25 \mathrm{FW}$ in our study may lie in its ability to measure walking speed, which has been shown to be a good predictor of falls in older populations (21). The 6MW, which is a test of endurance, had the least discriminatory value of all three tests. This may indicate that patients with ALD are not falling due to fatigue. Unlike the much longer $6 \mathrm{MW}$ test, the $25 \mathrm{FW}$ test can be easily administered in the clinic setting. Poor performance on this measure may alert clinicians to a patient's need for orthotics or physical therapy, which has shown to improve walking and reduce falls in patients with neuromuscular disease (22).

There are a few limitations of this study. First, given that the majority of our patient population was seeking neurological evaluation at an academic medical center, our cohort may overrepresent symptom burden in the broader ALD population. Second, the older age of females in our cohort likely contributes to our findings that gait and balance difficulties show no significant differences between sexes. Additionally, our finding 
that most falls during SOT occurred in conditions five and six may partly be explained by participant fatigue. Other limitations of this study include the lack of inter-rater reliability validation and the relatively small sample size of participants. While there are limitations to our method of scoring falls on balance testing, such as the lack of standardization of what constitutes a fall on the force plate, it may provide clinically relevant information for determining fall risk. Future work should include wireless technology such as motion sensors to detect subtle changes in head and trunk movements and characterize specific gait impairments not addressed in the current study.

In conclusion, we demonstrate that visual perturbations aggravate balance measures in both males and females with ALD, beyond the impact of the myelopathy characterized by functional gait analysis. Dynamic posturography highlights that the most impacted conditions depend on visual and vestibular input. By using technology that identifies and integrates somatosensory, vestibular, and visual information, we address the intricate balance control system impacted in ALD. Importantly, performance on all walking assessments was associated with a higher incidence of falls documented during CDP testing. This appeared independent of non-inflammatory brain lesions on MRI, pointing to the need for improved measures of balance and vestibular function in clinical practice.

\section{DATA AVAILABILITY STATEMENT}

The raw data supporting the conclusions of this article will be made available by the authors, without undue reservation.

\section{REFERENCES}

1. Mosser J, Douart A-M, Sarde C-O, Kioschisi P, Feil R, Moser H, et al. Putative $\mathrm{X}$-linked adrenoleukodystrophy gene shares unexpected homology with ABC transporters. Nature. (1993) 361:25. doi: 10.1038/361726a0

2. Powers JM, DeCiero DP, Ito M, Moser AB, Moser HW. Adrenomyeloneuropathy: a neuropathologic review featuring its noninflammatory myelopathy. J Neuropathol Exp Neurol. (2000) 59:89-102. doi: 10.1093/jnen/59.2.89

3. Engelen M, Barbier M, Dijkstra IM, Schür R, de Bie RM, Verhamme C, et al. $\mathrm{X}$-linked adrenoleukodystrophy in women: a cross-sectional cohort study. Brain. (2014) 137:693-706. doi: 10.1093/brain/awt361

4. Griffin JW, Goren E, Shaumburg H, Engel WK, Loriaux L. Adrenomyeloneuropathy: a probable variant of adrenoleukodystrophy I. Clinical and endocrinologic aspects. Neurology. (1977) 27:1107-13. doi: 10.1212/WNL.27.12.1107

5. van Geel BM, Bezman L, Loes DJ, Moser HW, Raymond GV. Evolution of phenotypes in adult male patients with X-linked adrenoleukodystrophy. Ann Neurol. (2001) 49:186-94. doi: 10.1002/1531-8249(20010201)49:2<186::AIDANA38>3.0.CO;2-R

6. Eichler F, Mahmood A, Loes D, Bezman L, Lin D, Moser H, et al. Magnetic resonance imaging detection of lesion progression in adult patients with X-linked adrenoleukodystrophy. Arch Neurol. (2007) 64:659-64. doi: 10.1001/archneur.64.5.659

7. Mallack EJ, van de Stadt S, Caruso PA, Musolino PL, Sadjadi R, Engelen M, et al. Clinical and radiographic course of arrested cerebral adrenoleukodystrophy. Neurology. (2020) 94:e2499-507. doi: 10.1212/WNL.0000000000009626

\section{ETHICS STATEMENT}

This study involving human participants was reviewed and approved by Mass General Brigham Human Research Committee. The patients/participants provided their written informed consent to participate in this study.

\section{AUTHOR CONTRIBUTIONS}

FE conceptualized and designed the study. NPG and RS performed the statistical analysis. NPG, RS, FE, CS, MB, and $\mathrm{RL}$ interpreted the data. NPG, MD, NRG, DK, PJ, and MB contributed substantially to the acquisition of data. NPG wrote the first draft of the manuscript. RS, MD, NRG, CS, MB, RL, and FE critically revised the manuscript for important intellectual content. All authors contributed to manuscript revision, read, and approved the submitted version.

\section{FUNDING}

FE receives research support from NINDS [R01 NS072446, R01 NS082331, U54 Global Leukodystrophy Initiative Clinical Trials Network (GLIA-CTN)], European Leukodystrophy Association, the Arrivederci Foundation, and the Leblang Foundation.

\section{ACKNOWLEDGMENTS}

We thank Paul Caruso and Otto Rapalino for radiological assessement of contrast enhancement on patient MRI scans.
8. van Geel BM, Poll-The BT, Verrips A, Boelens JJ, Kemp S, Engelen M. Hematopoietic cell transplantation does not prevent myelopathy in X-linked adrenoleukodystrophy: a retrospective study. J Inherit Metab Dis. (2015) 38:359-61. doi: 10.1007/s10545-014-9797-1

9. Keller JL, Wang JI, Kang JY, Hanson JA, Kamath P, Swain JO, et al. Strength: a relevant link to functional performance in the neurodegenerative disease of adrenomyeloneuropathy. Neurorehabil Neural Repair. (2012) 26:1080-8. doi: $10.1177 / 1545968312441682$

10. Huffnagel IC, van Ballegoij WJ, van Geel BM, Vos JM, Kemp S, Engelen M. Progression of myelopathy in males with adrenoleukodystrophy: towards clinical trial readiness. Brain. (2019) 142:334-43. doi: 10.1093/brain/awy299

11. van Ballegoij WJC, van de Stadt SIW, Huffnagel IC, Kemp S, van der Knaap MS, Engelen M. Postural body sway as surrogate outcome for myelopathy in adrenoleukodystrophy. Front Physiol. (2020) 11:786 doi: 10.3389/fphys.2020.00786

12. Nashner LM, Peters JF. Dynamic posturography in the diagnosis and management of dizziness and balance disorders. Neurol Clin. (1990) 8:331-49. doi: 10.1016/S0733-8619(18)30359-1

13. Nashner L, Jacobson G, Newman C, Kartush J. Handbook of Balance Function Testing. St. Louis, MO: Mosby Yearbook Inc. (1993). p. 88-9.

14. Bronte-Stewart HM, Minn AY, Rodrigues K, Buckley EL, Nashner LM. Postural instability in idiopathic Parkinson's disease: the role of medication and unilateral pallidotomy. Brain. (2002) 125:2100-14. doi: 10.1093/brain/awf207

15. Zammit G, Wang-Weigand S, Peng X. Use of computerized dynamic posturography to assess balance in older adults after nighttime awakenings using zolpidem as a reference. BMC Geriatr. (2008) 8:15. doi: 10.1186/1471-2318-8-15 
16. Kurtzke JF. Rating neurologic impairment in multiple sclerosis: an expanded disability status scale (EDSS). Neurology. (1983) 33:1444-52. doi: 10.1212/WNL.33.11.1444

17. Smart Equitest System Operator's Manual. Clackamas, OR: NeuroCom International, Inc. (2004).

18. Müller ML, Albin RL, Kotagal V, Koeppe RA, Scott PJ, Frey KA et al. Thalamic cholinergic innervation and postural sensory integration function in Parkinson's disease. Brain. (2013) 136:3282-9. doi: 10.1093/brain/a wt247

19. Rine RM, Schubert MC, Whitney SL, Roberts D, Redfern MS, Musolino $\mathrm{MC}$, et al. Vestibular function assessment using the NIH Toolbox. Neurology. (2013) 80(Suppl. 3):S25-31. doi: 10.1212/WNL.0b013e31828 72 c6a

20. Sebastião E, Sandroff BM, Learmonth YC, Motl RW. Validity of the timed up and go test as a measure of functional mobility in persons with multiple sclerosis. Arch Phys Med Rehabil. (2016) 97:1072-7. doi: 10.1016/j.apmr.2015.12.031

21. Howcroft J, Kofman J, Lemaire ED. Review of fall risk assessment in geriatric populations using inertial sensors. J Neuroeng Rehabil. (2013) 10:91. doi: 10.1186/1743-0003-10-91
22. Cup EH, Pieterse AJ, Jessica M, Munneke M, van Engelen BG, Hendricks HT, et al. Exercise therapy and other types of physical therapy for patients with neuromuscular diseases: a systematic review. Arch Phys Med Rehabil. (2007) 88:1452-64. doi: 10.1016/j.apmr.2007.07.024

Conflict of Interest: FE reports sponsored research contracts with Minoryx Therapeutics and consultancies with Autobahn Therapeutics, Minoryx Therapeutics, and SwanBio Therapeutics.

The remaining authors declare that the research was conducted in the absence of any commercial or financial relationships that could be construed as a potential conflict of interest.

Copyright (C) 2021 Godbole, Sadjadi, DeBono, Grant, Kelly, James, Stephen, Balkwill, Lewis and Eichler. This is an open-access article distributed under the terms of the Creative Commons Attribution License (CC BY). The use, distribution or reproduction in other forums is permitted, provided the original author(s) and the copyright owner(s) are credited and that the original publication in this journal is cited, in accordance with accepted academic practice. No use, distribution or reproduction is permitted which does not comply with these terms. 